\title{
Supporting Ebola Combat with Satellite Images: The MSF Perspective
}

\author{
Audrey Lessard-Fontaine ${ }^{1}$, Mathieu Soupart ${ }^{2}$ and Sylvie de Laborderie ${ }^{1}$ \\ ${ }^{1}$ CartONG Chambéry, France ·s_delaborderie@cartong.org \\ ${ }^{2}$ MSF GIS Unit ${ }^{1}$, Geneva/Switzerland
}

Short paper

\begin{abstract}
From the onset of the Ebola crisis, high resolution satellite imagery were used for digitizing roads, villages and towns, and to support base mapping of most affected areas. MSF's GIS unit requested the support of hundreds of volunteers of the OpenStreetMap community to manually digitize small subzones. The digitized data are available to be downloaded and used by GIS officers to support MSF work in the field with thematic maps as well as tailored base maps.
\end{abstract}

\section{Introduction}

In March 2014, cases of haemorrhagic fever were observed around the Guéckédou region in Guinea. Fearing the later confirmed possibility of an Ebola outbreak, MSF quickly deployed international staff to respond to the possible emergency. MSF-CH decided to deploy a dedicated Geographic Information Systems (GIS) officer along with the epidemiologist to offer support with up to date maps of the event. The decision to send a dedicated GIS officer to Guinea was informed by a study on the use of GIS within MSF (DE LABORDERIE et al. 2013) $)^{2}$, which had identified epidemiology as "the domain where GIS can bring the most positive evolution"3. As little geographic information was initially available, satellite imagery and crowdsourcing were largely used to gather base information (roads, buildings, villages, etc.) about the affected region. Specific GIS analysis could then be derived from this base information.

\section{Methodology}

The GIS officer initially focused on producing base maps of the affected region and its most important towns. At that point, only very basic maps existed of the prefecture of Guéckédou, which had been identified as the main field location for the Ebola response. Exist-

\footnotetext{
gisunit@geneva.msf.org

referred to as "GIS Study" in this document

3 GIS-Study, p. 34
}

GI Forum - Journal for Geographic Information Science, 1-2015.

(c) Herbert Wichmann Verlag, VDE VERLAG GMBH, Berlin/Offenbach. ISBN 978-3-87907-558-4.

(C) ÖAW Verlag, Wien. ISSN 2308-1708, doi:10.1553/giscience2015s445. 
ing maps listed main town locations, one major road, and also included low-quality paperdrawn city maps (Fig. 1).

Village locations were one of the main concerns since field trips to confirm the position of a village were necessary, but maps of major cities and roads in the region were also required to efficiently support the MSF teams. In order to quickly get more detailed maps of cities and major roads in the region, the GIS unit decided to acquire satellite imagery and crowdsource the task.

Satellite imagery was rapidly acquired with special web licence for three priority cities (Guéckédou, Macenta, and Kissidougou).

Through the volunteers of OpenStreetMap and the Humanitarian OpenStreetMap Team (HOT), satellite images that had been acquired by MSF-CH were turned into digital maps hosted on OpenStreetMap servers accessible to all volunteer mappers of the community so they could manually digitize the cities with high and very resolution satellite imagery as background maps. To ease the access to the satellite imagery web service, and to coordinate the work of dozens of volunteers, the OSM tasking mana-

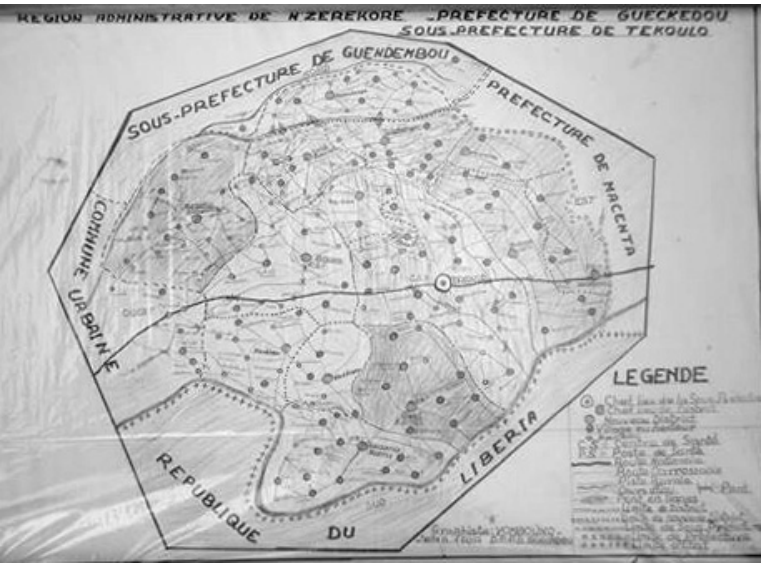

Fig. 1: Existing map of Guéckédou prior to the Emergency Onset

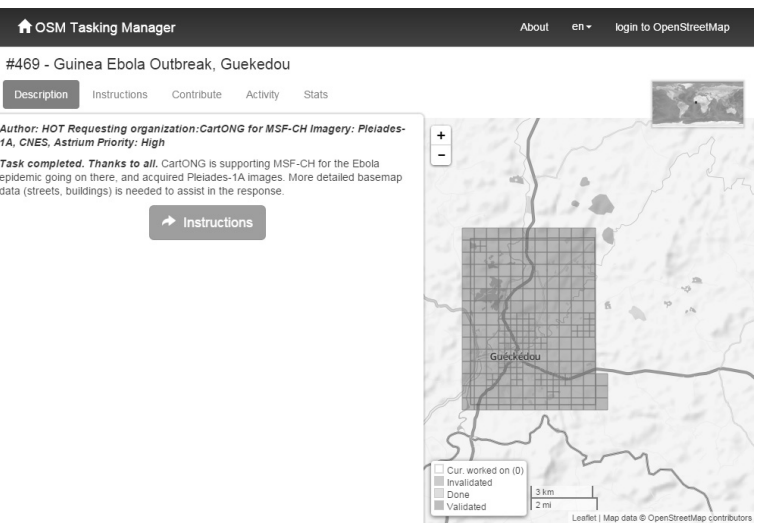

Fig. 2: OSM tasking manager for the digitalisation of Guéckédou, Guinea after the task completion ger was used (Fig. 2). This tool allows the volunteers to select a small subzone of the region of interest and digitize only the elements in that small zone in JOSM, an OpenStreetMap editor (Fig. 3). There is also a validation system in the tool, as advanced volunteers validate the work done by other volunteers. 

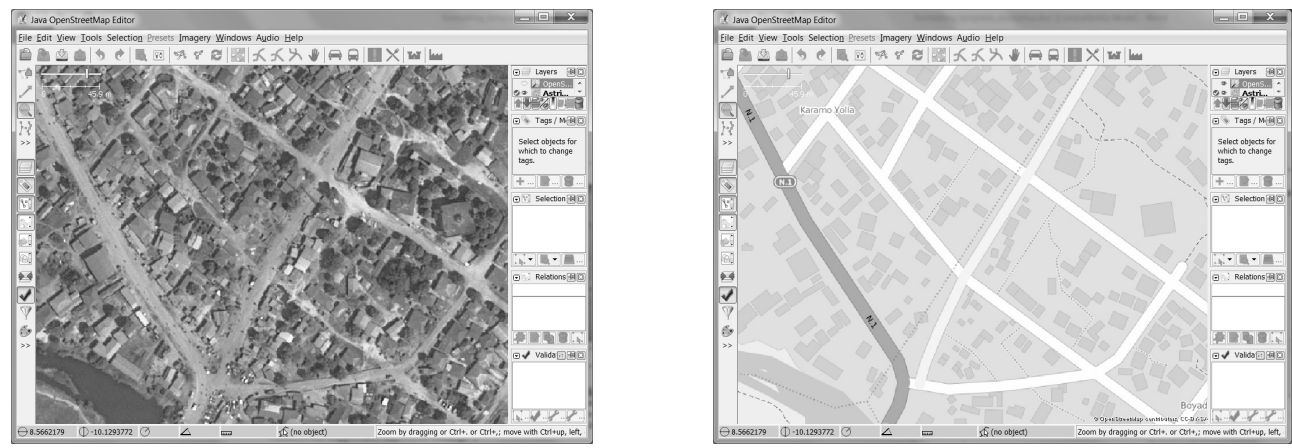

Fig. 3: View of JOSM: on the left, the very high resolution satellite imagery and on the right, the resulting map of the same zone

The three towns were mapped in less than three days. Within five days, 244 volunteers had mapped more than 90,000 buildings (Fig. 4).
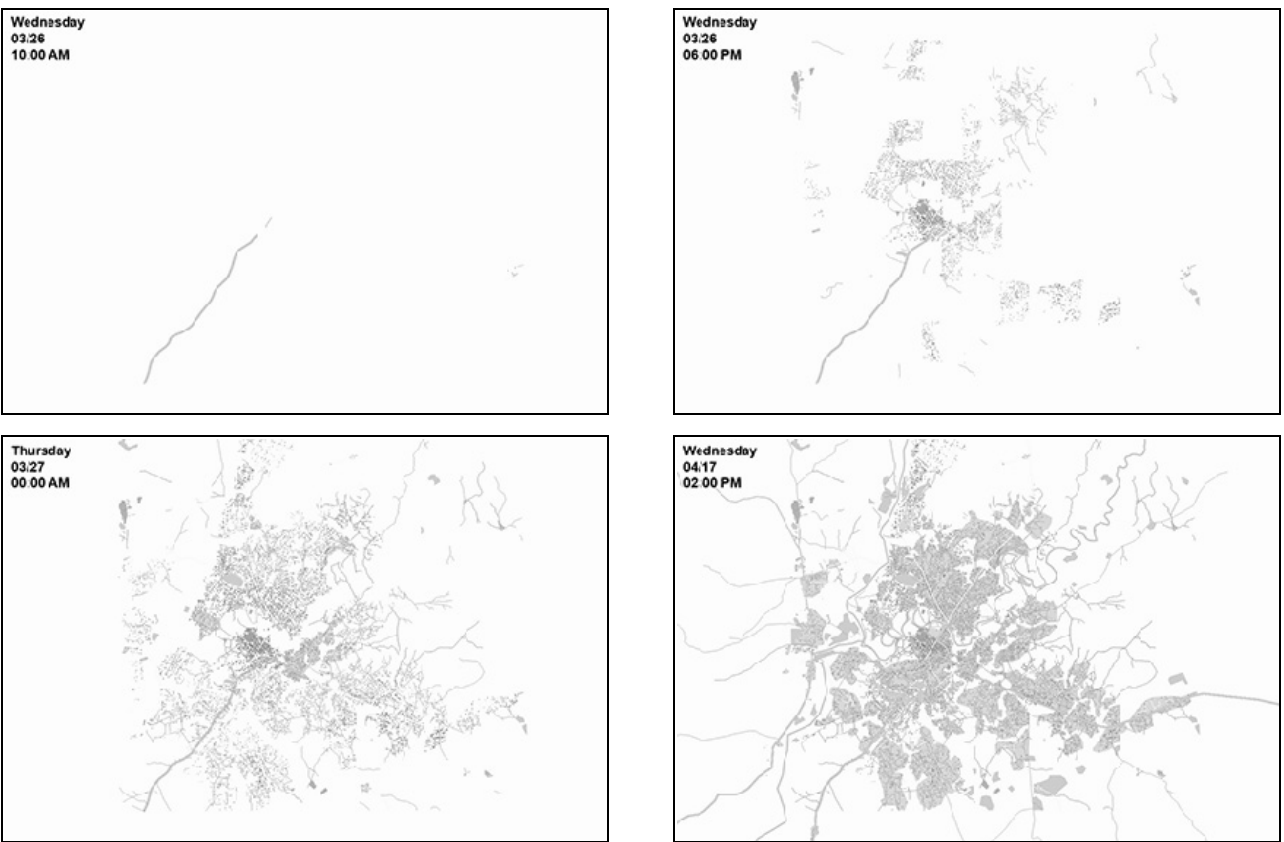

Fig. 4: Evolution of the Mapping of Guéckédou town within the first few hours of the activation, and a month later

As the crisis evolved, the OpenStreetMap community was able to access satellite imagery from different sources to provide the field with good base data of previously unmapped regions of all most affected countries (Guinea, Liberia, Sierra Leone). Most satellite imagery providers accepted to release imagery of the affected region for free, even though the 
International Charter on Space and Major Disasters ${ }^{4}$ had not been activated, which greatly helped the response timeliness. In the field, the base maps made available to MSF's operations through its Map Centre served as the foundation of many other map products such as epidemiologic maps to follow the epidemiology spread, logistics road access maps during the rainy season, etc. In fact, many of the internal MSF maps that were created by the GIS officer could not have been produced without these base maps. A large amount of work was also required in the field to confirm village names and positions as the respective databases were initially limited in the region, something that could not be done with the information available from the satellite imagery.

In the urban context such as in capital cities, satellite imagery was not only used to digitize information, but also served as background information for various thematic maps (Fig. 5). Indeed, in very dense environments, it was found useful to have very high-resolution information for the teams on the ground. The Disaster Charter activation (see above) later in the crisis made it possible for MSF and many other NGOs and governmental institutions to get quick and free access to satellite imagery over the whole region.

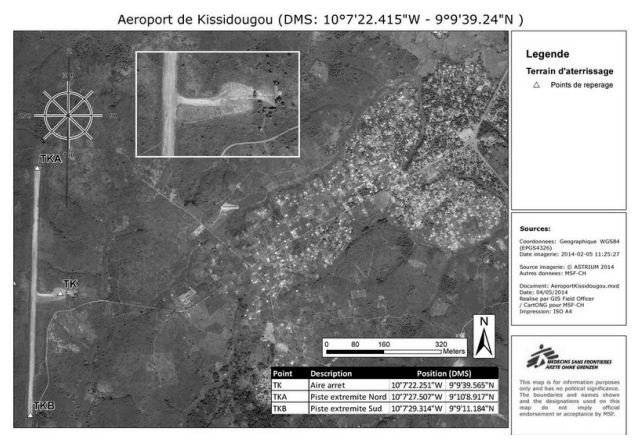

Fig. 5: Map using satellite imagery in urban context

\section{Conclusion}

Thanks to the digitization of large zones of the affected region from high and very highresolution satellite imagery ${ }^{5}$, the GIS officers in the field were able to quickly produce high quality basemaps, as well as various thematic maps in response to the need of the MSF teams (epidemiologic maps, log maps, communication maps, etc.). Coordination was the key to the collaborative work between satellite imagery providers, the HOT team, the OpenStreetMap community, and the MSF GIS unit to be efficient. It required a lot of energy from all parts, but allowed a quick cartographic support for the crisis. Very little advanced remote sensing analysis techniques were used in the crisis response context. Simple manual digitizing was found to be sufficiently efficient at this stage thanks to the crowdsourcing.

\section{References}

De Laborderie, S., Lessard-Fontaine, A. \& Soupart, M. (2013), State of art and opportunities using Geographic Information Systems in MSF. 82 p.

\footnotetext{
4 www.disasterscharter.org

5 Pléiades and SPOT- 6 but also Landsat
} 